\title{
Modes of HIV transmissions in Dhaka city
}

\author{
Mohammed N. Huq ${ }^{1}$, Saima Khan ${ }^{2}$, Wiwat Peerapatanapokin ${ }^{3}$, Masud Reza ${ }^{4}$, Zakir Hossain ${ }^{5}$, \\ Zubair Shams ${ }^{5}$, Md. A. Rahman ${ }^{6}$ \\ ${ }^{1}$ Department of Statistics, Jahangirnagar University, Savar, Dhaka, Bangladesh \\ ${ }^{2}$ UNAIDS Bangladesh \\ ${ }^{3}$ East-West Center \\ ${ }^{4}$ Center for HIV and AIDS, International Centre for Diarrhoeal Disease Research (ICDDR,B), Dhaka, Bangladesh \\ ${ }^{5}$ Save the Children \\ ${ }^{6}$ National AIDS/STD Programme
}

\begin{abstract}
Introduction: The epidemic in Dhaka, capital city of Bangladesh, was believed to be driven by injecting drug use as human immunodeficiency virus (HIV) has been concentrated among people who injected drugs since 2006. However, the needle/syringe program coupled with other prevention interventions among the key populations (KPs) and may have limited HIV spread from people who inject drugs (PWID) altering the modes of transmission (MOT).

Material and methods: The AIDS Epidemic Model was used to assess probable HIV transmission modes, which used data and information on indicators and co-factors from national behavioral surveys, serological surveillances, and other relevant studies including program data from 2000-2013.

Results: The distribution of HIV infections by MOT reveals that in 2000, needle sharing was the major source of HIV infection (48\%) in Dhaka, followed by (female) sex work (30\%), male-to-male sex (12\%), heterosexual relationships (husband to wife, 9\%), and other sources (1\%). In 2014, needle sharing as a mode of transmission dropped to $28 \%$. In the same year, male-to-male sex accounted for $42 \%$ of new HIV infections. It is projected that male-to-male sex will be responsible for 54 percent of new infections by 2020 in Dhaka.

Conclusions: The needle/syringe exchange program and other interventions among KPs have shifted the MOT in Dhaka city. Human immunodeficiency virus intervention strategies and coverage in Dhaka should be revised and focused to address the evolving epidemic.
\end{abstract}

HIV AIDS Rev 2017; 16, 2: 112-117 DOI: https://doi.org/10.5114/hivar.2017.66898

Key words: key populations, HIV new infections, mode of HIV transmission, AIDS epidemic model.

\section{Introduction}

In Bangladesh, like in other countries of the region, the human immunodeficiency virus (HIV) epidemic is still concentrated in key populations (sex workers, men who have sex with men, people who inject drugs, Hijra/transgender), particularly among people who inject drugs (PWID). Further, the epidemic is typically concentrated in major divisional cities. The national HIV prevalence rates among the key populations in 2011 were: PWID - 1\%, female sex workers (FSW) $-0.3 \%$, male sex workers (MSW) $-0 \%$, men who have sex with men (MSM) $-0 \%$ and Hijra $-1 \%$. However,
Address for correspondence: Dr. Mohammed N. Huq, Department of Statistics, Jahangirnagar University, Savar, 1342 Dhaka, Bangladesh, phone: +8801817549855 ,

e-mail: nhuq@juniv.edu
Article history:

Received: 9.08.2016

Received in revised form: 31.10 .2016

Accepted: 6.12.2016

Available online: 30.03 .2017
International Journal of HIV-Related Problems

HIV \& AIDS

R e v i e w 
in certain geographical areas, key populations have high HIV incidence, e.g. one neighborhood of Dhaka city accounted for $7.3 \%$ of HIV occurrence among PWID in 2007. The HIV prevalence among PWID in Dhaka has increased steadily over the years from $1.4 \%$ in 2000 to more than $7 \%$ in 2007; in 2011 it was $5.3 \%$ in this population group [1].

The first case of HIV in Bangladesh was detected in 1989, and till December 2014, the total number of reported cases was 3,674, of whom 563 have died, leaving 3,111 reported people living with HIV (PLHIV). However, the majority of infections are likely to remain undetected, and the total national estimate is over 9,500 PLHIV [2]. The National AIDS/STD Programme (NASP) also confirmed 433 new HIV infections in 2015, of which 124 (28.6\%) new HIV cases were reported in Dhaka city alone [3].

The HIV epidemic in Dhaka is believed to be largely driven by injecting drug use. However, the presence of several high-risk groups in Dhaka has resulted in a more complex local HIV epidemic among PWID than elsewhere in the country. The high prevalence of HIV infections among the PWID in Dhaka city is considered to have the potential of spreading the epidemic among PWID, and this can spread into the general population $[4,5]$.

Recent information indicates that the risk behaviors, such as sharing of injecting equipment and a decline in consistent condom use in sexual encounters between PWID and female sex workers, enhances the spread of the HIV infection in Dhaka city [6]. A recent study undertaken by International Centre for Diarrhoeal Disease Research (ICDDR,B) in 2011 among male injecting drug users indicated that nearly $44.2 \%$ of the PWID share needles and syringes. The study also documents that the average number of injections per day was 2.4. The percent of male PWID who visit female sex workers was estimated at $21.9 \%$. Although a significant portion of the male injecting drug users reported to use condom during their last sex with sex workers, condom use with their regular partners or spouses was reported to be $27.7 \%$ [7] The evidence also shows that some female drug users in Bangladesh turn to sex work out of financial necessity to support their addiction [8].

Further, the frequency of pre-marital and extra-marital sex and the large number of sexual acts with sex workers are commonly known factors associated with the transmission of HIV in Bangladesh $[9,10]$. Among the general population, approximately $10 \%$ of men reported having bought sex from female sex workers [11]. A national survey among youth conducted in 2008 also reports that almost $20 \%$ of unmarried males reported having premarital sex and for $28 \%$ of these respondents, the last sex was with a sex worker. The reported consistent condom use with female sex workers among this group was $48 \%$ in 2008 [6].

A behavioral survey conducted among MSM in Dhaka indicated that $71 \%$ of MSM reported having anal sex with commercial or non-commercial male partners during the past one month, and only $26 \%$ reported to have used condoms during their last anal sex [9]. In addition, 30 percent of the MSM bought sex from female sex workers and another $46.7 \%$ pur- chased sex services from male sex workers in the last one month preceding the survey [12].

A recent behavioral survey by ICDDR,B conducted in 2010 among Hijras in Dhaka reported that most Hijra (87.5\%) had anal sex with male partners in the last month, and only $19.4 \%$ used a condom during the last sex act [13].

Along with the risk behaviors of the key populations, migration is also widely recognized as a potential factor in facilitating HIV transmission in Bangladesh due to an engagement in unsafe, casual, or commercial sex, which potentially increases the risk of exposure to HIV. According to NASP, 47 of the 259 cases of PLHIV between 2002 and 2004 were linked to migration. Of these, 29 were males returning from abroad, 7 were wives of migrant workers, and 4 were children of HIV-positive migrant workers. In 2004, data from NASP showed that 57 of the 102 newly reported HIV cases were among returning migrants [14].

In the current global financial climate, it is more important than ever that effective targeting of program response as well as resource allocation for the control of HIV infection is based on informed, strategic decision-making. Planning HIV prevention programs requires up-to-date information on the likely sources of new infections and a framework for understanding epidemic patterns and for highlighting priority areas for prevention [15-17]. Under the circumstance, the aim of the study is to understand the HIV transmission dynamics and epidemiological trends in Dhaka city and inform the policy makers for designing effective intervention strategies.

\section{Material and methods}

The AIDS Epidemic Model (AEM) was applied to assess the likely pattern of HIV spread in Dhaka based on current risk indicators and HIV prevalence. The AEM is a semi-empirical process model that can reflect various dynamics driving HIV transmission. The user adjusts AEM fitting parameters and co-factors until HIV prevalence outputs from the model agree with observed epidemiological trends. When a parameter is changed, AEM recalculates the epidemic and adjusts the estimates. These parameters in the model are set on a country-specific basis based upon fitting local epidemiological data. It also allows the user to examine changes in the dominant modes of transmission over time [18].

AIDS Epidemic Model is patterned after the dominant modes of HIV transmission in Asian settings and uses semi-empirical fits to actual country data; thus, the resulting projections using behavioral, serological, and program data from Dhaka are likely to reflect the actual HIV transmission patterns in this city.

Once the trends in risk behaviors among the important sub-populations in Dhaka are used as inputs, and the model determines the HIV transmission probabilities necessary to fit observed epidemiological patterns, as seen in surveillance data, it can then calculate the number of new infections through key routes of transmission including marital 
sex, extra-marital (casual) sex, sex work, male-male sex, needle sharing, and mother-to-child transmission. AIDS Epidemic Model produces large variety of outputs including transmission routes, male/ female ratios, AIDS cases, and HIV trends, and allows numerous opportunities for external validation of the projections produced against other sources of data.

The population groups included in the model are divided into ten compartments: i) males who are clients of sex workers; ii) males who are not clients of sex workers; iii) lower risk general female population ; iv) FSW with higher risk behavior; v) FSW with lower risk behavior; vi) PWID in higher risk sharing networks; vii) PWID who are in lower risk networks or do not share; viii) MSM, who are not sex workers; ix) MSWs; and x) Hijra (transgender).

Among the several output indicators of the model, the study uses the estimates for HIV prevalence, number of people living with HIV, and number of new HIV infections in Dhaka between 2000 and 2020. For model analysis, each compartment is divided into those infected with HIV and those not infected. Movement is allowed between compartments as people do not remain in one category until death. Movement between or out of compartments may also occur due to deaths or infection with HIV. Infection occurs either through sexual behavior or needle sharing with an infected partner. The number of new infections is calculated based on the occurrence in the partner population, the frequency of sex or injecting acts, and the probability of transmission of HIV. Corrections are made for increased HIV transmission in the presence of other STIs or due to lack of male circumcision by adding co-factors that increase the effective transmission probability by a fixed amount. Finally, a correction is made for protective behaviors such as using condoms [19].

For example, the number of male clients infected by sex workers in a year is calculated as:

\begin{tabular}{|l|l|}
\hline $\begin{array}{l}\text { Number of new } \\
\text { infections in male clients } \\
\text { from sex workers }\end{array}$ & $=\begin{array}{l}\text { Probability of female to } \\
\text { male transmission per } \\
\text { contact } x \text { number of client } \\
\text { contacts per year with sex } \\
\text { workers } \times \text { fraction that are } \\
\text { not protected by condoms } \\
\times \text { percent of sex workers } \\
\text { infected } \times \text { corrections } \\
\text { for other STIs and for } \\
\text { ircumcision }\end{array}$ \\
\hline
\end{tabular}

In order to estimate the number of people living with HIV (PLHIV) and HIV prevalence in Dhaka city, the model specifies that HIV prevalence at any time is a function of the number of new infections that have arisen prior to that time, multiplied by the probability of surviving of the infected individuals.

The updated version software and documentation are available on the EastWest Center (www.EastWestCenter. org) and Avenir Health (www.avenirhealth.org) websites.

\section{Data requirements}

AIDS Epidemic Model uses time series data on several behavioral indicators and HIV prevalence data points for every key population, which are obtained from both surveillance systems and surveys. The data used for the analysis are summarized below:

- size of these key populations (expressed as a percentage of adult males or females 15-49) and of the adult male and female population as a whole,

- average duration, for which people remain in key populations expressed in years, and used to calculate turnover and movement between key populations,

- frequency of risk behaviors - e.g., the number of vaginal or anal intercourse acts per week and/or number of injections in the last year,

- levels of protective measures taken with different partner types - e.g., condom use between sex workers and clients, reducing the fraction of injections shared or the prevalence of sharing,

- HIV and STI prevalence - accounted for in each key population,

- data on adult ART i.e., number of adults receiving ART by gender,

- information on program coverage among key populations and program unit cost.

Data from multiple sources are collected for the period of 2000-2013 and used in this study. The main data sources include: Bangladesh Bureau of Statistics, National Serological and Behavioral Surveillance reports, assessments and operations research conducted on sex work, male sexual behavior, most at risk adolescents, harm reduction, etc., National Syndromic Management Guidelines, size estimations and counting of key populations, Bangladesh Demographic and Health Surveys as well as program coverage data and case reports by the National AIDS/STD Program of Ministry of Health and Family Welfare.

Some of the information/data requirements were based on assumptions agreed on by a broader technical stakeholder group, as there were data gaps for some indicators such as IDU mortality, mobility between key population groups, client make-up, male circumcision, etc. Also, HIV prevalence data for Hijra was adjusted assuming that the available data was only for those who are sex workers.

\section{Results}

\section{HIV prevalence among key populations in Dhaka city}

The HIV prevalence among PWID (males) in Dhaka showed an upward trend from 2000 to 2007 (Fig. 1). The prevalence was estimated at less than $1 \%$ in 2000 and reached the peak at $7.1 \%$ in 2007 . Thereafter, it appears that the prevalence rate among PWID (males) has stabilized. In 2014, the occurrence among this group was estimated to be around 6\%. With the assumption that the current intervention programs will continue to sustain current behavior 


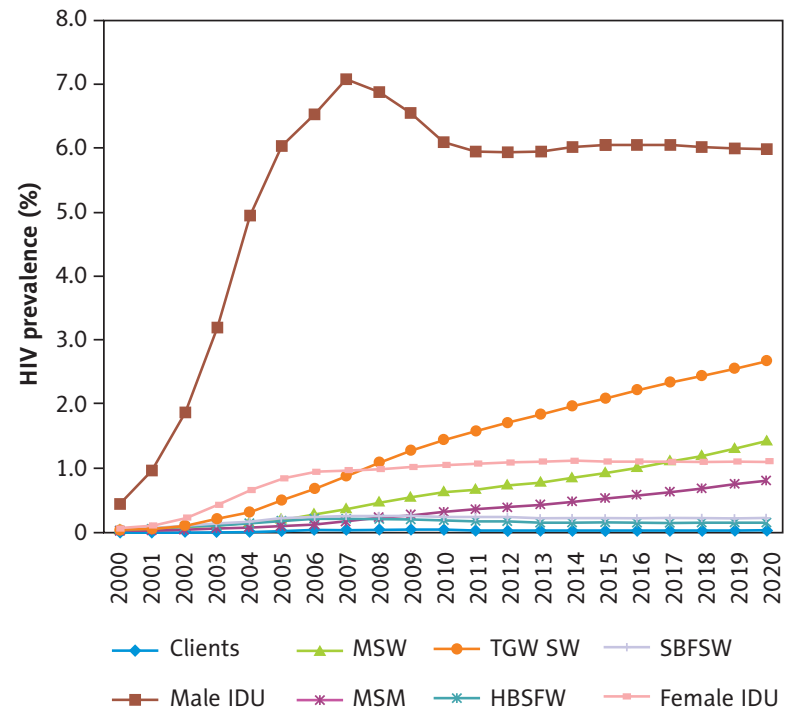

Figure 1. Trends of HIV prevalence in Dhaka city if interventions continue

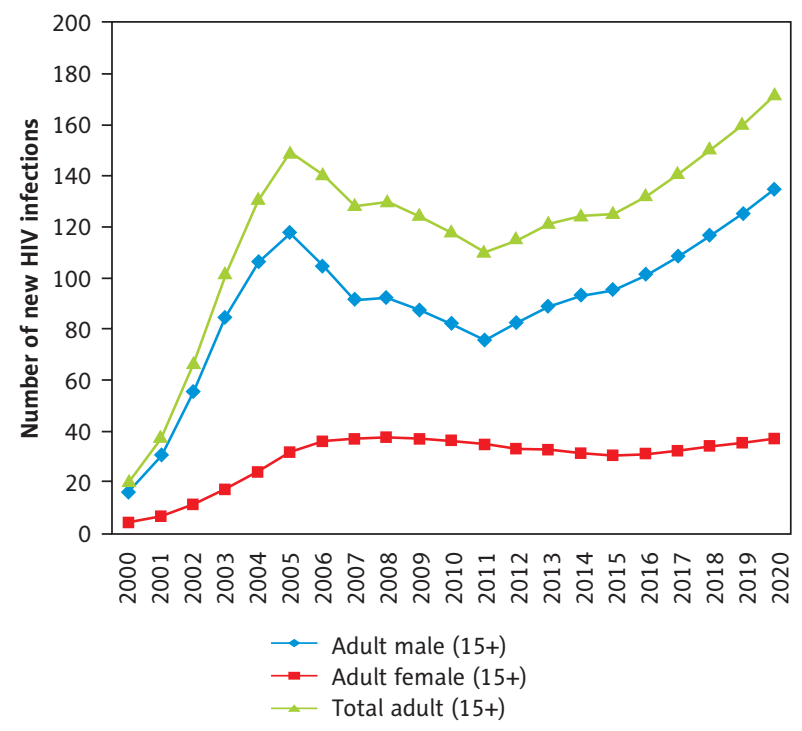

Figure 3. Trends in new HIV infections in adult population

trends, the projected prevalence will remain fairly stable at about 6\% among PWID (males) in Dhaka between 2014 and 2020. The general trend for PWID (females) was a rise in the prevalence of HIV to $1.1 \%$ in 2014 , as compared to less than $0.1 \%$ in 2000 . From 2014 and onwards, the prevalence among PWID (females) is projected to remain stable at approximately $1.1 \%$, if interventions continue.

The distribution of HIV prevalence among other key populations is also presented in Figure 1. The HIV occurrence among FSW is estimated at $0.2 \%$ in 2014 and will remain stable at this point over the projection period. On the other hand, HIV prevalence among Hijra sex workers (TG-SW), MSM, and MSW demonstrates a steady increasing trend over the period. It is estimated that the HIV prevalence among Hijra sex workers is $2.0 \%$ in 2014, compared

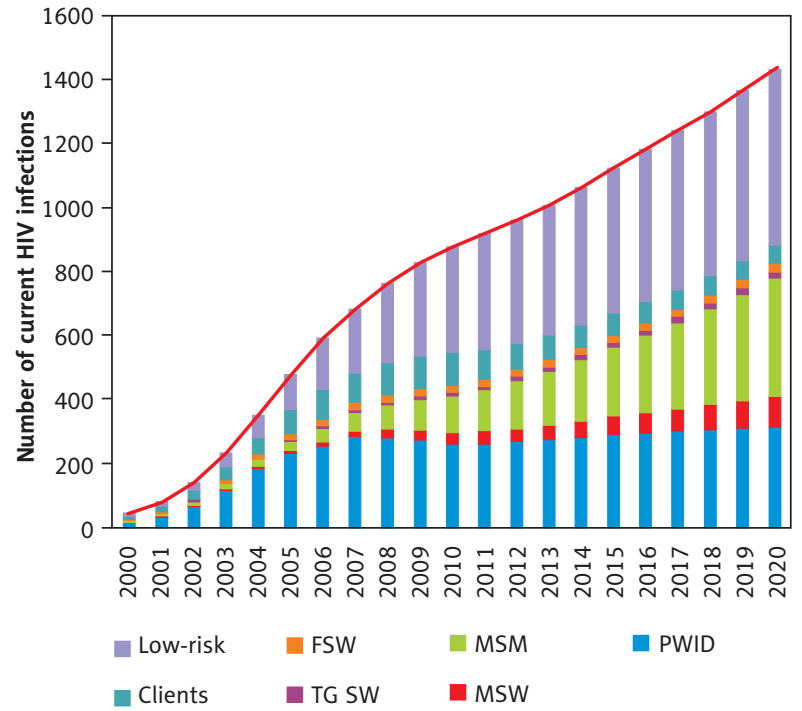

Figure 2. Number of current HIV infections by sub-population

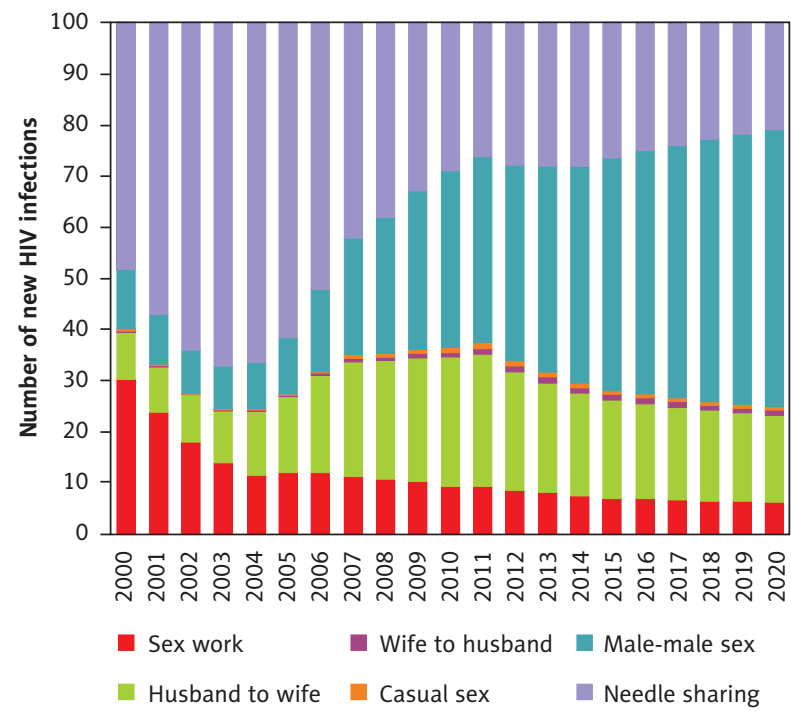

Figure 4. New HIV infections by mode of transmission

to less than $0.1 \%$ in 2000 . HIV occurrence among Hijra sex workers will reach $2.4 \%$ in 2020 . In addition, HIV prevalence among MSW and MSM was estimated at $0.9 \%$ and $0.5 \%$ in 2014 , respectively. The same rate of occurrence will reach $1.4 \%$ among MSW and $0.8 \%$ among MSM in 2020. Provided intervention coverage remains stable to sustain the current behaviors in terms of needle sharing, condom use, management of sexually transmitted infections, etc.

\section{People currently living with HIV in Dhaka city}

The number of PLHIV (15+ years) in Dhaka city is estimated to be 1,061 in 2014 as compared to only 45 in 2000, representing more than 23 -fold increase over the period 
(Figure 2). The number of PLHIV in Dhaka might reach 1,436 by 2020 , even with interventions ongoing. Spreading of people living with HIV by different key population groups reveals that in 2014, 281 PWID were living with HIV in Dhaka, and this number should remain fairly stable until 2020. In contrast, the amount of current HIV infections will significantly increase among the MSM (93\%) between 2014 and 2020. Moreover, the number of current infections among low risk people will increase to 551 in 2020, as compared to 428 in 2014.

\section{Number of new HIV infections in Dhaka city}

The estimated number of new infections among adults (15+ years) in Dhaka has increased from 20 in 2000 to 149 in 2005 , representing more than a seven-fold increase in only five years (Figure 3 ). The number then started to decrease slightly, falling to 115 in 2012. It is estimated that the number of new HIV infections in Dhaka city will eventually start to increase in the coming years. The number of new infections among adults is estimated to be 124 in 2014 (male - 93 and female - 31), with a male-female ratio of 3.0. The number of new infections is representing a sharp increase between 2014 and 2020 among the male adults (45\%), as compared to their female counterpart (18\%).

\section{New HIV infections by mode of transmission in Dhaka city}

The distribution of new HIV infections by mode of transmission (Figure 4) reveals that in 2000 needle sharing was the major source of HIV infection (48\%) among the adult population in Dhaka city, followed by (female) sex work (30\%), male-to-male sex (12\%), heterosexual relationship among spouses (husband to wife, 9\%), and other modes (1\%). The source of HIV infections shows a different pattern in 2014, with a sharp drop in the proportion due to needle sharing ( $48 \%$ in 2000 vs. $28 \%$ in 2014 ). In the same year, the proportion of new infections due to unprotected maleto-male sex has significantly increased from $12 \%$ in 2000 to $42 \%$ in 2014. Moreover, in 2014, husband-to-wife transmission stands at $20 \%$, followed by female sex work at $7 \%$, and casual sex from wife to husband represents $3 \%$ of new infections. It is projected that male-to-male sex will account for $54 \%$ of the new infections, and needle sharing will be responsible for $21 \%$ by the year 2020 in Dhaka.

\section{Discussion}

The estimated number of new infections among adults (15+ years) in Dhaka city has been increasing over the last 15 years. During 2000-2006, the HIV epidemic in Dhaka was largely driven by needle sharing due to injecting drug use $[4,5,7,14]$. Nevertheless, the enhanced harm reduction interventions contributed significantly in containing of the HIV epidemic among the PWID in Dhaka [14].
The study findings also reveal that needle sharing was the major source of HIV infection (48\%) in Dhaka city in 2000 , and the proportion of new infections due to needle sharing decreased to $28 \%$ in 2014 . This is most probably linked to the impact of the harm reduction interventions among PWID in Dhaka city. Consequently, the source of new infections in Dhaka has been changed over the last one decade. The proportion of new HIV infections due to male-to-male sex in Dhaka city has significantly increased from $12 \%$ in 2000 to $42 \%$ in 2014 . Moreover, the proportion of new infections from male-to-male sex had gradually increased from 2014, and would represent a major source of new HIV infections in the coming years, as 54 out of 100 new HIV infections in Dhaka in 2020 would be accounted for by MSM behavior.

The study results also provide evidence, which suggests that the number of new HIV infections among key populations particularly among MSM and MSW, will continue to increase in Dhaka city even if the existing intervention coverage remains stable to sustain the current behaviors in terms of needle sharing, condom use, management of sexually transmitted infections, etc. The implication would not be only to continue current interventions in Dhaka, but also increase program coverage among MSM and MSW. Also, the need to identify more cases through HTC is necessary, as a prime way of preventing HIV transmission in Dhaka. Further, it would also be interesting to understand the MOT in the rest of the nation to help future interventions planning.

Human immunodeficiency virus estimation and modes of transmissions derived from the AEM are essential to the assessment of the HIV situation in different key populations, thereby keeping intervention activities and future plans focused on priority areas $[14,18,20]$. It is anticipated that the study findings will contribute meaningfully in planning HIV response in the country with particular focus in Dhaka city. However, the study suffers from few limitations. For example, after 2006, a sufficient data on behaviors was not available for Dhaka, or at national scale across covered and uncovered key populations. The gaps were overcome by using data available from various sub-national studies, using AEM default values and certain assumptions in discussion with technical experts, etc.

\section{Conclusions}

The new HIV infections in the coming years will be increasing in Dhaka city through various high-risk behaviors, particularly through male-to-male sex. Thus, geographically focused specific prevention efforts are needed in reducing the spread of the epidemic. The focus maybe different in various geographical regions, and in Dhaka, it seems that current interventions should continue, but not through business as usual strategies. To prevent the related consequences of increasing new HIV infections by the year 2020, case detection needs to increase among key population groups with subsequent linkage to care and support services to prevent further spread of HIV. Furthermore, MSM and MSW needs 
an increased intervention coverage along with the strengthened HIV testing and counseling (HTC).

This enhanced understanding could strengthen the capacity to respond to HIV among key populations before the epidemic worsens. The study suggests continued and increased attention to the problem, as investing now will be a much more cost-effective way of achieving sustainable program goals than waiting until the epidemic takes its full toll on communities and the country.

\section{Conflict of interest}

The author's declared no potential conflicts of interest with respect to the research, authorship, and/or publication of this article.

\section{References}

1. National AIDS/STD Program. National HIV Serological Surveillance, 2011 Bangladesh: 9th Round Technical Report. Ministry of Health and Family Welfare, Directorate General of Health Services, NASP. Dhaka, 2011.

2. UNAIDS, Global AIDS response progress reporting 2014. Joint United Nations Programme on HIV/AIDS. Geneva, 2014.

3. National AIDS/STD Program (NASP). National AIDS statistics unveiled on International AIDS Day. Ministry of Health and Family Welfare, Directorate General of Health Services, NASP. Dhaka, 2013.

4. Azim T, Alam MS, Rahman M, et al. Impending concentrated HIV epidemic among injecting drug users in Central Bangladesh. Int J STD AIDS 2004; 15: 280-282.

5. Azim T, Hussein N, Kelly R. Effectiveness of harm reduction programs for injecting drug users in Dhaka city. Harm Reduc J 2005; 2: 22.

6. Ministry of Health and Family Welfare (MOHFW)/Save the Children and ICDDR,B. Endline HIV/AIDS Survey among Youth in Bangladesh. MOHFW, Directorate General of Health Services, National AIDS/STD Program (NASP). Dhaka, 2008.

7. International Centre for Diarrhoeal Disease Research, Bangladesh (ICDDR,B). Assessing the effectiveness and quality of harm reduction services for injecting drug users delivered through their peers in Dhaka and identifying gaps and barriers to effective service provision. Report Submitted to Save the Children, USA, December 2011.

8. Hossain KT, Habib SE, Imam H. Commercial Sexual Behavior and Risk Factors in Bangladesh: An Investigation of Female Sex Workers in Bangladesh. University of Rajshahi, Department of Sociology/DFID: Higher Education Link Program, 2004.

9. National Institute of Population Research and Training (NIPORT), Mitra and Associates, and Macro International. Bangladesh Demographic and Health Survey 2007. Dhaka, Bangladesh and Calverton, Maryland, USA: NIPORT, Mitra and Associates, and Macro International, 2009.

10. Habib SE, Amanullah, ASM, Lovejoy F, et al. Risking the future: unprotected intercourse and AIDS knowledge among female commercial sex workers in central Bangladesh. International Quarterly of Community Health Education 2000-2001; 20: 265-280.

11. Chowdhury ME, et al. Assessment of sexual behavior of men in Bangladesh: A Methodological Experiment. FHI/ICDDR,B, Dhaka 2006.

12. ICDDR,B. Risk of and vulnerability to HIV among MSM in Dhaka, Bangladesh: A behavioral survey of MSM in Dhaka. ICDDR,B, Dhaka 2011.

13. ICDDR,B. Risk of and vulnerability to HIV among Hijra in Dhaka, Bangladesh: A behavioral survey of Hijra in Dhaka. ICDDR,B, Dhaka 2011.
14. National AIDS/STD Program (NASP). Assessment of impact of harm reduction interventions among people who inject drugs (PWID) in Dhaka City. Dhaka, Bangladesh: Ministry of Health and Family Welfare, Directorate General of Health Services, NASP, 2014.

15. Case KK, Ghys PD, Gouws E, et al. Understanding the modes of transmission model of new HIV infection and its use in prevention planning. Bulletin of the World Health Organization 2012; 90: 831-838A.

16. Gouws E, White PJ, Stover J, Brown T. Short term estimates of adult HIV incidence by mode of transmission: Kenya and Thailand as examples. Sex Transm Infect 2006; 82: iii51-55.

17. New HIV infections by mode of transmission in West Africa: a multi-country analysis. UNAIDS Regional Support Team for West and Central Africa, Dakar 2010.

18. Brown T, Peerapatanapokin W. The Asian Epidemic Model: a process model for exploring HIV policy and programme alternatives in Asia. Sex Transm Infect 2004; 80 (Suppl I): i19-i24.

19. Ghys PD, Brown T, Grassly NG, et al. The UNAIDS Estimation and Projection Package: a software package to estimate and project national HIV epidemics. Sex Transm Infect 2004; 80 (Suppl I): i5-9.

20. Reddy A, Brown T. The Asian Epidemic Model for Dhaka City 2006: The Consequences of Current Risk. FHI Bangladesh, Analysis and Advocacy Project, Dhaka 2008. 